\title{
Collective oscillations of a classical gas confined in harmonic traps
}

\author{
David Guéry-Odelin ${ }^{1,2}$, Francesca Zambelli ${ }^{1}$, Jean Dalibard ${ }^{2}$, Sandro Stringari ${ }^{1}$ \\ ${ }^{1}$ Dipartimento di Fisica, Università di Trento, \\ and Istituto Nazionale per la Fisica della Materia, I-38050 Povo, Italy \\ ${ }^{2}$ Laboratoire Kastler Brossel, Ecole normale supérieure \\ 24, Rue Lhomond, F-75231 Paris Cedex 05, France
}

(October 10, 2018)

\begin{abstract}
Starting from the Boltzmann equation we calculate the frequency and the damping of the monopole and quadrupole oscillations of a classical gas confined in an harmonic potential. The collisional term is treated in the relaxation time approximation and a gaussian ansatz is used for its evaluation. Our approach provides an explicit description of the transition between the hydrodynamic and collisionless regimes in both spherical and deformed traps. The predictions are compared with the results of a numerical simulation.
\end{abstract}

PACS numbers: 03.75.Fi, 05.30.Jp, 67.40.Db

\section{INTRODUCTION}

After the experimental realization of Bose-Einstein condensation in trapped atomic gases [1], the investigation of collective excitations in these systems has become a very popular subject of research (see [2] for a recent theoretical review). At very low temperature, when the the whole system is Bose-Einstein condensed, the motion is described by the hydrodynamic equations of superfluids. These equations, which can be directly derived starting from the mean field Gross-Pitaevskii equation for the order parameter, give predictions [3] in very good agreement with experiments [4]. At higher temperatures the mean field effects become less important, while collisional terms cannot be longer ignored. If the temperature is notably larger than the critical temperature for Bose-Einstein condensation the dynamical behaviour of a dilute gas is well described by the Boltzmann equation. In this case two different regimes may occur: a collisional (hydrodynamic) regime characterized by conditions of local statistical equilibrium and a collisionless regime where the motion is described by the single particle hamiltonian. Differently from the case of uniform gases, also in the collisionless regime the system exhibits well defined oscillations which are driven by the external confinement. The equations for the collisional regime were investigated in [5.6], while a phenomenological interpolation between the two regimes was proposed in [7].

The purpose of this paper is to provide an analysis of the lowest oscillation modes (eigenfrequency and damping) using the classical Boltzmann equation. In particular we study the transition between the hydrodynamic and collisionless regimes. Our approach is based on the relaxation time approximation for the collisional integral.
The corresponding relaxation time is explicitly calculated by means of a gaussian ansatz for the distibution function which allows for an analytic evaluation of the collisional contribution to the equations of motion.

For harmonic trapping the gaussian ansatz exactly reproduces the solution of the classical Boltzmann equation in both the collisional and collisionless regimes and is consequently expected to be a good approximation also in the intermediate regime. This is explicitly checked by comparing the analytic predictions of the gaussian ansatz with the exact results of a numerical simulation based on molecular dynamics.

In our paper atoms behave like hardspheres, $\sigma_{0}$ being their total cross section which will be assumed to be energy independent. This is well satisfied in classical ultracold gases where collisions are completely characterized by the $s$-wave scattering length and the cross section is thus isotropic and in most cases energy independent.

\section{METHOD OF AVERAGES}

The starting point of our analysis is the Boltzmann equation for the phase space distribution function $f(\mathbf{r}, \mathbf{v}, t)$ 9]:

$$
\frac{\partial f}{\partial t}+\mathbf{v} \cdot \nabla_{\mathbf{r}} f+\frac{\mathbf{F}}{m} \cdot \nabla_{\mathbf{v}} f=I_{\text {coll }}(f)
$$

where

$$
\begin{aligned}
I_{\mathrm{coll}}\left(f\left(\mathbf{r}, \mathbf{v}_{\mathbf{1}}\right)\right)= & \frac{\sigma_{0}}{4 \pi} \int d^{2} \Omega d^{3} v_{2}\left|\mathbf{v}_{2}-\mathbf{v}_{1}\right| \\
& {\left[f\left(\mathbf{v}_{1}^{\prime}\right) f\left(\mathbf{v}_{2}^{\prime}\right)-f\left(\mathbf{v}_{1}\right) f\left(\mathbf{v}_{2}\right)\right] }
\end{aligned}
$$

is the usual classical collisional integral.

It accounts for elastic collisions between particles 1 and 2 , with initial velocities $\mathbf{v}_{1}$ et $\mathbf{v}_{2}$, and final velocities $\mathbf{v}_{1}^{\prime}$ and $\mathbf{v}_{2}^{\prime}$. The solid angle $\Omega$ gives the direction of the final relative velocity. The expression for the collisional term can be easily extended to include effects of both Bose and Fermi statistics [8]. Actually most of the results discussed in this paper hold also in the presence of quantum degeneracy, provided the system is not BoseEinstein condensed and one can ignore mean field effects. The quantitative estimates of collisional effects will be however based on classical statistics. 
The force $\mathbf{F}_{\text {trap }}=-\nabla U_{\text {trap }}(x, y, z)$ is produced by the confining potential which in the following will be chosen to be of harmonic form:

$$
U_{\text {trap }}(x, y, z)=\frac{1}{2} m \omega_{\perp}^{2}\left(x^{2}+y^{2}\right)+\frac{1}{2} m \omega_{z}^{2} z^{2} .
$$

We introduce the anisotropy parameter $\lambda=\omega_{z} / \omega_{\perp}$. For $\lambda=1$, one deals with an isotropic harmonic trap, for $\lambda^{2} \ll 1$ one has a cigar shaped trap and for $\lambda^{2} \gg 1$ a disk shaped trap. Starting from (11), one can derive useful equations for the average of a general dynamical quantity $\chi(\mathbf{r}, \mathbf{v})$ :

$$
\frac{d\langle\chi\rangle}{d t}-\left\langle\mathbf{v} \cdot \nabla_{\mathbf{r}} \chi\right\rangle-\left\langle\frac{\mathbf{F}_{\text {trap }}}{m} \cdot \nabla_{\mathbf{v}} \chi\right\rangle=\left\langle\chi I_{\text {coll }}\right\rangle,
$$

where the average is taken in both position and velocity space:

$$
\langle\chi\rangle=\frac{1}{N} \int d^{3} r d^{3} v f(\mathbf{r}, \mathbf{v}, t) \chi(\mathbf{r}, \mathbf{v}),
$$

The quantity $\left\langle\chi I_{\text {coll }}\right\rangle$ can be written in the useful form

$$
\left\langle\chi I_{\text {coll }}\right\rangle=\frac{1}{4 N} \int d^{3} r d^{3} v_{1}\left[\chi_{1}+\chi_{2}-\chi_{1^{\prime}}-\chi_{2^{\prime}}\right] I_{\text {coll }}(f)
$$

as a consequence of the invariance properties of the cross section (we have put $\left.\chi_{i}=\chi\left(\mathbf{r}, \mathbf{v}_{i}\right)\right)$. The collisional contribution (5) is equal to zero if $\chi$ corresponds to a dynamic quantity conserved during the elastic collision. This happens if $\chi$ can be written in the form 9, 10

$$
\chi=a(\mathbf{r})+\mathbf{b}(\mathbf{r}) \cdot \mathbf{v}+c(\mathbf{r}) \mathbf{v}^{2} .
$$

\section{MONOPOLE OSCILLATION IN HARMONIC ISOTROPIC TRAPS}

Let us consider an harmonic isotropic trapping potential $\left(\omega_{x}=\omega_{y}=\omega_{z} \equiv \omega_{0}\right)$. As a first application of (3), one can immediately derive the behaviour of the monopole mode 10 12 by computing the evolution of the square radius:

$$
\frac{d\left\langle\mathbf{r}^{2}\right\rangle}{d t}=2\langle\mathbf{r} . \mathbf{v}\rangle
$$

In order to obtain a closed set of equations one also needs the following equations

$$
\frac{d\langle\mathbf{r} \cdot \mathbf{v}\rangle}{d t}=\left\langle\mathbf{v}^{2}\right\rangle-\omega_{0}^{2}\left\langle\mathbf{r}^{2}\right\rangle
$$

and

$$
\frac{d\left\langle\mathbf{v}^{2}\right\rangle}{d t}=\omega_{0}^{2}\left\langle\mathbf{r}^{2}\right\rangle
$$

force and for the energy. The collisional term does not contribute to the above equations because all the dynamic quantities satisfy the criterium (6). So there is no damping for the "breathing" mode of a classical dilute gas confined in an harmonic isotropic trap. The same is true if one includes quantum degeneracy effects in the collisional term of the Boltzmann equation.

By looking for solutions of eqs.(7) evolving in time as $e^{i \omega t}$ one immediately finds the result $\omega=2 \omega_{0}$, holding for all collisional regimes from the collisionless to the hydrodynamical one. The occurrence of this monopole undamped solution was first pointed out by Boltzmann (see, for example, the discussion in [1]]).

It is worth noticing that the frequency of the classical monopole oscillation in isotropic harmonic traps differs from the one of a Bose-Einstein condensed gas at $T=0$. In the latter case the monopole oscillation is still undamped, but the frequency, for large $N$, is $\omega=\sqrt{5} \omega_{0}$ [3]. The difference is the consequence of the mean field interaction which is absent in the Boltzmann equation (11). Furthermore, at finite temperature the monopole oscillation is expected to exhibit damping because of the coupling between the condensate and the thermal component of the gas.

\section{QUADRUPOLE OSCILLATION AND RELAXATION TIME APPROXIMATION}

The purpose of this section is to investigate the quadrupole mode of a classical gas as well as its coupling with the monopole oscillation arising in anisotropic traps. In this case the solution of the Boltzmann equation exhibits damping and one has to deal explicitly with the collisional term. In the presence of anistropy, the $l_{z}=0$ component of the quadrupole is coupled with the monopole and one finally finds the following set of coupled equations :

$$
\begin{gathered}
\frac{d\left\langle\chi_{1}\right\rangle}{d t}-2\left\langle\chi_{3}\right\rangle=0 \\
\frac{d\left\langle\chi_{2}\right\rangle}{d t}-2\left\langle\chi_{4}\right\rangle=0 \\
\frac{d\left\langle\chi_{3}\right\rangle}{d t}-\left\langle\chi_{5}\right\rangle+\frac{2 \omega_{\perp}^{2}+\omega_{z}^{2}}{3}\left\langle\chi_{1}\right\rangle+\frac{\omega_{z}^{2}-\omega_{\perp}^{2}}{3}\left\langle\chi_{2}\right\rangle=0 \\
\frac{d\left\langle\chi_{4}\right\rangle}{d t}-\left\langle\chi_{6}\right\rangle+\frac{2 \omega_{z}^{2}-2 \omega_{\perp}^{2}}{3}\left\langle\chi_{1}\right\rangle+\frac{\omega_{\perp}^{2}+2 \omega_{z}^{2}}{3}\left\langle\chi_{2}\right\rangle=0 \\
\frac{d\left\langle\chi_{5}\right\rangle}{d t}+\frac{2 \omega_{z}^{2}+4 \omega_{\perp}^{2}}{3}\left\langle\chi_{3}\right\rangle+\frac{2 \omega_{z}^{2}-2 \omega_{\perp}^{2}}{3}\left\langle\chi_{4}\right\rangle=0 \\
\frac{d\left\langle\chi_{6}\right\rangle}{d t}+\frac{4 \omega_{z}^{2}-4 \omega_{\perp}^{2}}{3}\left\langle\chi_{3}\right\rangle+\frac{4 \omega_{z}^{2}+2 \omega_{\perp}^{2}}{3}\left\langle\chi_{4}\right\rangle=\left\langle\chi_{6} I_{\text {coll }}\right\rangle
\end{gathered}
$$


where we have defined the quantities

$$
\begin{aligned}
& \chi_{1}=\mathbf{r}^{2} \\
& \chi_{2}=2 z^{2}-r_{\perp}^{2} \\
& \chi_{3}=\mathbf{r} \cdot \mathbf{v} \\
& \chi_{4}=2 z v_{z}-\mathbf{r}_{\perp} \cdot \mathbf{v}_{\perp} \\
& \chi_{5}=\mathbf{v}^{2} \\
& \chi_{6}=2 v_{z}^{2}-v_{\perp}^{2} .
\end{aligned}
$$

If the trap is isotropic $\left(\omega_{\perp}=\omega_{z}\right)$, (10) decouples in two subsystems. One subsystem refers to the undamped monopole oscillations discussed in the previous section. The other corresponds to the damped quadrupole mode. Notice that collisions affects only the last equation of (10). Actually only the variable $\chi_{6}=2 v_{z}^{2}-v_{\perp}^{2}$ is not a conserved quantity and hence does not satisfy the criterium (6). The collisional contribution can be estimated by treating the collisional integral in the so-called relaxation time approximation 10$]$ :

$$
I_{\text {coll }}(f)=-\frac{f-f_{\ell . e .}}{\tau}
$$

where $\tau$ is a relaxation time. As we will see in the next section this time can be explicitly calculated in terms of the scattering cross section. The function $f_{\ell . e}$. entering (12) is the local equilibrium distribution function and is characterized by the same density, velocity field and local temperature as the distribution function $f$. Differently from $f$ it has, however, an isotropic velocity distribution.

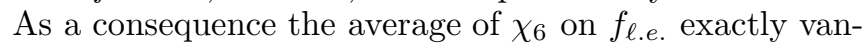
ishes and one can write

$$
\left\langle\chi_{6} I_{\text {coll }}\right\rangle=-\frac{\left\langle\chi_{6}\right\rangle}{\tau}
$$

The system (10) is now linear, and one can search for solutions of the type $e^{i \omega t}$ and deduce the associated determinant. The result is:

$$
\begin{array}{r}
\left(\omega^{2}-4 \omega_{z}^{2}\right)\left(\omega^{2}-4 \omega_{\perp}^{2}\right)- \\
\frac{i}{\omega \tau}\left(\omega^{4}-\frac{2}{3} \omega^{2}\left(5 \omega_{\perp}^{2}+4 \omega_{z}^{2}\right)+8 \omega_{\perp}^{2} \omega_{z}^{2}\right)=0
\end{array}
$$

The first term of (14) corresponds to the dispersion law for the pure collisionless regime $\left(\omega_{z} \tau \longrightarrow \infty\right)$. In this case the eigenfrequencies coincide with the ones predicted by the single particle harmonic oscillator hamiltonian: $\omega_{\mathrm{CL}}=2 \omega_{z}$ and $\omega_{\mathrm{CL}}=2 \omega_{\perp}$. On the contrary, the term multiplying $1 / \tau$ refers to the pure hydrodynamical regime $\left(\omega_{z} \tau \longrightarrow 0\right)$.

For the spherical trap, one gets $\omega_{\mathrm{HD}}=\sqrt{2} \omega_{0}$ and $2 \omega_{0}$ for the quadrupole and monopole modes respectively. For a cigar shaped configuration $\left(\lambda^{2} \ll 1\right)$ the two hydrodynamic solutions have the form $\omega_{\mathrm{HD}}=\sqrt{12 / 5} \omega_{z}$ and $\sqrt{10 / 3} \omega_{\perp}$ [5], while for a disk trap $\left(\lambda^{2} \gg 1\right)$ one finds $\omega_{\mathrm{HD}}=\sqrt{8 / 3} \omega_{z}$ and $\sqrt{3} \omega_{\perp}$.
Formula (14), which provides the proper interpolation between the collisionless and hydrodynamic regimes, can be simplified in the case of a spherical, cigar, and disk shaped trap. In fact, the dispersion law (14) can be written in all these limiting cases in the useful form:

$$
\omega^{2}=\omega_{\mathrm{CL}}^{2}+\frac{\omega_{\mathrm{HD}}^{2}-\omega_{\mathrm{CL}}^{2}}{1+i \omega \tilde{\tau}},
$$

typical of relaxation phenomena $[7,13]$. The time $\tilde{\tau}$ is related to $\tau$ by a simple numerical factor. For example $\tilde{\tau}=\tau$ for the quadrupole mode in the spherical case, and $\tilde{\tau}=6 \tau / 5$ for the lowest mode of the cigar shaped configuration. A relevant feature of $(15)$ is the presence of an imaginary part, associated with the damping of oscillations. By writing the frequency as $\omega=\omega_{r}+i \Gamma$ one finds, assuming $\Gamma \ll \omega_{r}$,

$$
\Gamma \simeq \frac{\tilde{\tau}}{2} \frac{\omega_{\mathrm{CL}}^{2}-\omega_{\mathrm{HD}}^{2}}{1+\left(\omega_{r} \tilde{\tau}\right)^{2}} .
$$

Notice that the damping depends crucially on the difference between the frequencies calculated in the collisional and hydrodynamic regimes and exactly vanishes when these frequencies coincide. This happens, for example, in the monopole case for isotropic trapping, as discussed in the previous section 14 .

In the hydrodynamic limit $\left(\omega_{r} \tilde{\tau} \rightarrow 0\right)$ the damping predicted by (16) takes the form

$$
\Gamma_{\mathrm{HD}} \simeq \frac{\tilde{\tau}}{2}\left(\omega_{\mathrm{CL}}^{2}-\omega_{\mathrm{HD}}^{2}\right),
$$

while in the opposite regime $(\tau \rightarrow \infty)$ one gets

$$
\Gamma_{\mathrm{CL}} \simeq \frac{1}{2 \omega_{\mathrm{CL}}^{2} \tilde{\tau}}\left(\omega_{\mathrm{CL}}^{2}-\omega_{\mathrm{HD}}^{2}\right)
$$

A maximum for $\Gamma$ is found at $\omega_{r} \tilde{\tau} \sim 1$, leading to $\Gamma \sim$ $\left(\omega_{\mathrm{CL}}^{2}-\omega_{\mathrm{HD}}^{2}\right) / \omega_{r}$. Around this value, the approximation leading to (16) is no longer accurate, and one should rather use (14) or (15).

\section{GAUSSIAN ANSATZ}

One expects the relaxation time $\tau$ to be of the same order of the inverse of the collision rate $\gamma_{\text {coll }}$ [15]:

$$
\gamma_{\text {coll }}=\frac{n(0) v_{\mathrm{th}} \sigma_{0}}{2}
$$

giving the number of collisions undergone by a given atom per unit of time. In (19) $v_{\text {th }}=\sqrt{8 \theta_{0} / \pi m}$ is the thermal velocity and $n(0)$ the central density.

An explicit evaluation of this link can be obtained by making a gaussian ansatz for the distribution function, which allows for an analytic evaluation of the collisional contribution to the equations of motion. In fact, a natural assumption to describe the $l_{z}=0$ modes characterized by axial symmetry is given by the parametrization 


$$
\begin{gathered}
f(\mathbf{r}, \mathbf{v}, t)=N\left(\frac{m}{2 \pi}\right)^{3} \frac{\gamma_{\perp} \gamma_{z}^{1 / 2}}{\theta_{\perp} \theta_{z}^{1 / 2}} \\
e^{-m U_{\perp}^{2} / 2 \theta_{\perp}} e^{-m U_{z}^{2} / 2 \theta_{z}} e^{-m\left(\gamma_{\perp} r_{\perp}^{2}+\gamma_{z} z^{2}\right) / 2}
\end{gathered}
$$

where $r_{\perp}=\sqrt{x^{2}+y^{2}}$ and $\mathbf{U}=\mathbf{v}-\langle\mathbf{v}\rangle$. Eq. (20) provides a natural generalization of the local equilibrium distribution, by introducing a deformation not only in coordinate space (taken into account by the $\gamma$ parameters), but also in velocity space. The gaussian ansatz (20) can be shown to describe exactly the monopole and quadrupole oscillations both in the hydrodynamic and collisionless regimes. In the former case, the velocity distribution is isotropic and hence $\theta_{\perp}=\theta_{z}$. For the collisionless case, an exact solution of the equations of motion can be found in the form (20). However, in this case, $\theta_{\perp}$ is different from $\theta_{z}$, corresponding to configurations far from local equilibrium. Only in the presence of isotropic trapping and for the monopole oscillation the hydrodynamic and collisionless solutions coincide. In this case the ansatz (20), with $\theta_{\perp}=\theta_{z}$ and $\gamma_{\perp}=\gamma_{z}$ provides an exact solution of the Boltzmann equation.

In the limit of small oscillations around the equilibrium configuration, the temperature in axial and transverse axis can be expanded around the equilibrium temperature $\theta_{0}$ :

$$
\begin{aligned}
\theta_{\perp} & =\theta_{0}+\delta \theta_{\perp} \\
\theta_{z} & =\theta_{0}+\delta \theta_{z}
\end{aligned}
$$

One then finds:

$$
\left\langle\chi_{6}\right\rangle=\frac{2}{m}\left(\delta \theta_{z}-\delta \theta_{\perp}\right)
$$

showing that $\left\langle\chi_{6}\right\rangle$ is, as expected, directly sensitive to the anisotropy of the velocity distribution. The collisional contribution to the equation for $\chi_{6}$ (last eq. of the system (10)), can be also expressed in terms of this anisotropy. By inserting eq. (20) into eq. (5) with $\chi=\chi_{6}$ we obtain, after linearization, the expression

$$
\begin{gathered}
\left\langle\chi_{6} I_{\text {coll }}\right\rangle=\frac{1}{32 \pi} \frac{m \sigma_{0}}{N \theta_{0}^{2}} \int d^{3} r d^{3} U_{1} d^{3} U_{2}\left|\mathbf{U}_{1}-\mathbf{U}_{2}\right| d^{2} \Omega f_{0}(1) \\
f_{0}(2) \Delta \chi_{6}\left[\delta \theta_{\perp}\left(\left(U_{\perp}^{2}\right)_{1^{\prime}}+\left(U_{\perp}^{2}\right)_{2^{\prime}}-\left(U_{\perp}^{2}\right)_{1}-\left(U_{\perp}^{2}\right)_{2}\right)+\right. \\
\left.\delta \theta_{z}\left(\left(U_{z}^{2}\right)_{1^{\prime}}+\left(U_{z}^{2}\right)_{2^{\prime}}-\left(U_{z}^{2}\right)_{1}-\left(U_{z}^{2}\right)_{2}\right)\right],
\end{gathered}
$$

where $f_{0}$ is the gaussian (20) evaluated at equilibium, and $\Delta \chi_{6}=\left(\chi_{6}\right)_{1}+\left(\chi_{6}\right)_{2}-\left(\chi_{6}\right)_{1^{\prime}}-\left(\chi_{6}\right)_{2^{\prime}}$. After some length but straightforward algebra (see Appendix) the integral (23) can be written in the useful form:

$$
\left\langle\chi_{6} I_{\mathrm{coll}}\right\rangle=-\frac{2}{\tau} \frac{\left(\delta \theta_{z}-\delta \theta_{\perp}\right)}{m}=-\frac{\left\langle\chi_{6}\right\rangle}{\tau},
$$

with $\tau$ given by

$$
\tau=\frac{5}{4 \gamma_{\mathrm{coll}}} .
$$

Eq. 25) provides the explicit link between $\gamma_{\text {coll }}$ and the relaxation time for the quadrupole $l_{z}=0$ mode. Notice that this relationship, provided by the gaussian ansatz (20), is independent of the deformation of the trap. In the next section we will compare this prediction with the result of a numerical calculation based on molecular dynamics.

\section{NUMERICAL SIMULATION}

In this section, we present results for the dispersion law arising from a numerical analysis, based on a molecular dynamics simulation. We consider $N=2 \times 10^{4}$ particles moving in the potential (2). Binary elastic collisions are taken into account using a boxing technique [16, 17. At each time step $\delta t$, the position of each particle is discretized on a square lattice with a step $\xi$. The volume $\xi^{3}$ of a box is chosen such that the average occupation $p_{\text {occ }}$ of any box is much smaller than 1 . Collisions occur only between two particles occupying the same box, and the time step $\delta t$ is adjusted in such a way that the probability $p_{\text {coll }}$ of a collisional event during $\delta t$ is also much smaller than 1 . We choose typically $p_{\text {occ }} \sim p_{\text {coll }} \sim 5 \%$.

Initial conditions for exciting the lowest energetical mode are obtained by a deformation in coordinate and velocity spaces of the cloud along the weak axes, keeping the phase space density constant. We have checked that this method leads to the excitation of only the lowest frequency mode. Then, we let the cloud evolve. The damped oscillation of the dynamical variable $\chi_{2}=$ $2 z^{2}-r_{\perp}^{2}$ is analysed for different choices of the collision rate. As an example, the oscillation frequency and the damping for a cigar shaped trap $(\lambda=1 / 10)$ are plotted (solid circles) respectively on figures (1) and (2). One observes that the frequency decreases as the ratio $\omega_{z} / \gamma_{\text {coll }}$ decreases and tends asymptotically to the hydrodynamic value $\sqrt{12 / 5} \omega_{z}=1.55 \omega_{z}$. For large value of $\omega_{z} / \gamma_{\text {coll }}$, the frquency instead approaches the collisionless value $2 \omega_{z}$. By performing a least square fit with formula (14), we obtain:

$$
\tau=(1.31 \pm 0.07) \frac{1}{\gamma_{\text {coll }}}
$$

which well agree with the gaussian prediction 25). The full line on figure (1) and (2) corresponds to eq. (14) with $\tau$ given by the gaussian ansatz prediction (25). We have checked that, for deformed cigar traps $(\lambda \ll 1)$, result (26) is independent of the value of $\lambda$.

We have instead found that, as the deformation parameter $\lambda$ becomes larger, the results of the numerical simulation exhibits an increasing deviation from the gaussian prediction. A discrepancy of the order of $25 \%$ has been 
observed for $\lambda=\sqrt{8}$. The physical origin of this deviation remains to be undestood. In all cases, the gaussian ansatz underestimates the coefficient of proportionality between the relaxation time $\tau$ and the inverse of the collision rate $1 / \gamma_{\text {coll }}$.

\section{CONCLUSION}

In this work, we have presented an investigation of the collective frequencies of a classical gas trapped in an harmonic potential well. Starting from the classical Boltzmann equation, we have derived a set of coupled equations for the averages of the relevant dynamic variables associated with the monopole and quadrupole modes. The collisional term has been treated in the time relaxation approximation. The corresponding relaxation time was evaluated by a gaussian ansatz for the distribution function. The quality of the gaussian prediction has been checked numerically by a numerical simulation based on molecular dynamics.

\section{APPENDIX A: COLLISIONAL INTEGRAL}

The appendix is devoted to the explicit calculation of the collisional integral (23). Let us introduce the center of mass velocity $\mathbf{C}$ and the relative velocity before $(\mathbf{V})$ and after $\left(\mathbf{V}^{\prime}\right)$ collision :

$$
\begin{aligned}
\mathbf{U}_{1} & =\mathbf{C}+\mathbf{V} / 2 \\
\mathbf{U}_{2} & =\mathbf{C}-\mathbf{V} / 2 \\
\mathbf{U}_{1^{\prime}} & =\mathbf{C}+\mathbf{V}^{\prime} / 2 \\
\mathbf{U}_{2^{\prime}} & =\mathbf{C}-\mathbf{V}^{\prime} / 2
\end{aligned}
$$

The conservation of kinetic energy during an elastic collision ensures

$$
V^{2}=V^{\prime 2}
$$

so that the collisional integral can be rewritten in the form

$$
\begin{array}{r}
\left\langle\chi_{6} I_{\text {coll }}\right\rangle=-\left(\delta \theta_{z}-\delta \theta_{\perp}\right) \frac{3}{128 \pi} \frac{m \sigma_{0}}{N \theta_{0}^{2}} \\
\int d^{3} r d^{3} V d^{3} C d^{2} \Omega V f_{0}(1) f_{0}(2)\left[V_{z}^{2}-V_{z^{\prime}}^{2}\right]^{2} .
\end{array}
$$

Let us first calculate the angular integral :

$$
I_{\Omega} \equiv \int d \Omega\left[V_{z}^{2}-V_{z}^{\prime 2}\right]^{2}
$$

We introduce a reference frame $\Re$ linked to $\mathbf{V}$, the vectors of the associated orthonormal basis beeing $(\hat{\mathbf{a}}, \hat{\mathbf{b}}, \hat{\mathbf{c}})$. Without loss of generality we choose $\hat{\mathbf{a}}$ such that $\mathbf{V}=$ $V \cdot \hat{\mathbf{a}}$, and the $z$ axis in the plane generated by $(\hat{\mathbf{a}}, \hat{\mathbf{b}})$. The relative velocity $\mathbf{V}^{\prime}$ is characterized in $\Re$ by two spherical angles $\left(\theta^{\prime}, \varphi^{\prime}\right)$ :

$$
\begin{aligned}
\mathbf{V}^{\prime} \cdot \hat{\mathbf{a}} & =V \cos \theta^{\prime} \\
\mathbf{V}^{\prime} \cdot \hat{\mathbf{b}} & =V \sin \theta^{\prime} \cos \varphi^{\prime} \\
\mathbf{V}^{\prime} \cdot \hat{\mathbf{c}} & =V \sin \theta^{\prime} \sin \varphi^{\prime}
\end{aligned}
$$

Thus

$$
V_{z}^{\prime}=\mathbf{V}^{\prime} \cdot \hat{\mathbf{z}}=V_{z} \cos \theta^{\prime}+V \sin \theta^{\prime} \cos \varphi^{\prime}(\hat{\mathbf{b}} \cdot \hat{\mathbf{z}})
$$

where $\hat{\mathbf{z}}$ is the unit vector of the $z$ axis and

$$
\begin{aligned}
V_{z}^{\prime 2} & =V_{z}^{2} \cos ^{2} \theta^{\prime}+V^{2} \sin ^{2} \theta^{\prime} \cos ^{2} \varphi^{\prime}(\hat{\mathbf{b}} \cdot \hat{\mathbf{z}})^{2} \\
& +2 V V_{z} \cos \theta^{\prime} \sin \theta^{\prime} \cos \varphi^{\prime}(\hat{\mathbf{b}} \cdot \hat{\mathbf{z}})
\end{aligned}
$$

With our choice of coordinate, one has

$$
\hat{\mathbf{z}} \cdot \hat{\mathbf{z}}=1=(\hat{\mathbf{a}} \cdot \hat{\mathbf{z}})^{2}+(\hat{\mathbf{b}} \cdot \hat{\mathbf{z}})^{2},
$$

which implies

$$
V^{2}(\hat{\mathbf{b}} \cdot \hat{\mathbf{z}})^{2}=V^{2}-V_{z}^{2}
$$

and, finally,

$$
\begin{aligned}
V_{z}^{2}-V_{z}^{\prime 2} & =V_{z}^{2}\left(1-\cos ^{2} \theta^{\prime}\right)-\sin ^{2} \theta^{\prime} \cos ^{2} \varphi^{\prime}\left(V^{2}-V_{z}^{2}\right) \\
& 2 V V_{z} \sin \theta^{\prime} \cos \theta^{\prime} \cos \varphi^{\prime}(\hat{\mathbf{b}} \cdot \hat{\mathbf{z}})
\end{aligned}
$$

By integrating the square of the previous expression, one finds

$$
I_{\Omega}=\frac{32 \pi}{15}\left(\frac{15}{8} V_{z}^{4}+\frac{3}{8} V^{4}-\frac{5}{4} V_{z}^{2} V^{2}\right) .
$$

The calculation of the collisional integral (A3) is now straightforward, and finally yields the result :

$$
\left\langle\chi_{6} I_{\mathrm{coll}}\right\rangle=-\left(\delta \theta_{z}-\delta \theta_{\perp}\right) \frac{4}{5 m} v_{\mathrm{th}} \sigma_{0} n(0),
$$

where $v_{\text {th }}=\sqrt{8 \theta_{0} / \pi m}$ is the thermal velocity of a particle of the gas. A9 permits to derive (24,25) with $\gamma_{\text {coll }}$ defined by eq. (19).

[1] M.H. Anderson, J.R. Ensher, M.R. Matthews, C.E. Wieman, and E.A. Cornell, Science 269,198 (1995); K.B. Davis, M.-O. Mewes, M.R. Andrews, N.J. van Druten, D.S. Durfee, D.M. Kurn, and W. Ketterle, Phys. Rev. Lett. 75, 3969 (1995).

[2] F. Dalfovo, S. Giorgini, L.P. Pitaevskii, and S. Stringari, Reviews of Modern Physics, 71 (1999).

[3] S. Stringari, Phys. Rev. Lett., 77, 2360 (1996). 
[4] M.-O. Mewes, M.R. Andrews, N.J. van Druten, D.M. Kurn, S.D. Durfee, S. Inouye, and W. Ketterle, Phys. Rev. Lett., 77, 416 (1996); D.S. Jin, J.R. Ensher, M.R. Matthews, C.E. Wieman, and E.A. Cornell, Phys. Rev. Lett., 77, 420 (1996); M.R. Andrews, D.M. Kurn, H.-J. Miesner, D.S. Durfee, C.G. Townsend, S. Inouye, and W. Ketterle, Phys. Rev. Lett., 79, 553 (1997) and Erratum (1998), Phys. Rev. Lett., 80, 2967; U. Ernst, A. Marte, F. Schreck, J. Schuster, and G. Rempe, Europhys. Lett. 41, 1 (1998).

[5] A. Griffin, Wen-Chin Wu, and S. Stringari, Phys. Rev. Lett. 78, 1838 (1997).

[6] Y. Kagan, E.L. Surkov and G. Shlyapnikov, Phys. Rev. Lett. 79, 2604 (1997).

[7] G.M. Kavoulakis, C.J. Pethick, and H. Smith, Phys. Rev. A 57, 2938, (1998).

[8] L.P. Kadanoff and G. Baym, Quantum Statistical Mechanics (W.A. Benjamin, New York, 1962).

[9] K. Huang, Statistical Mechanics, (J. Wiley, New York, 1987), 2nd ed.

[10] G.E. Uhlenbeck and G.W. Ford, Lectures in Statistical Mechanics, (American Mathematical Society, Providence, R.I., 1963).

[11] C. Cercignani, The Boltzmann equation and its applications, (Springer Verlag, New York, 1988).

[12] L. Boltzmann, in Wissenschaftliche Abhandlungen, edited by F. Hasenorl (J.A. Barth, Leipzig, 1909), Vol II, p. 83.

[13] L.D. Landau and E.M. Lifshitz, §81, Fluid Mechanics, (Pergamon Press, Oxford, 1987).

[14] Another important case where the damping identically vanishes is the center of mass (dipole) oscillation, whose frequency coincides with the one of the harmonic potential, in both hydrodynamic and collisionless regimes.

[15] H. Wu, E. Arimondo and C.J. Foot, Phys. Rev. A, 56, 560 (1997).

[16] H. Wu and C.J. Foot, J. Phys. B:At. Mol. Opt. Phys., 29, L321 (1996).

[17] D. Guéry-Odelin, J. Söding, P. Desbiolles and J. Dalibard, Optics Express 2, 323 (1998).

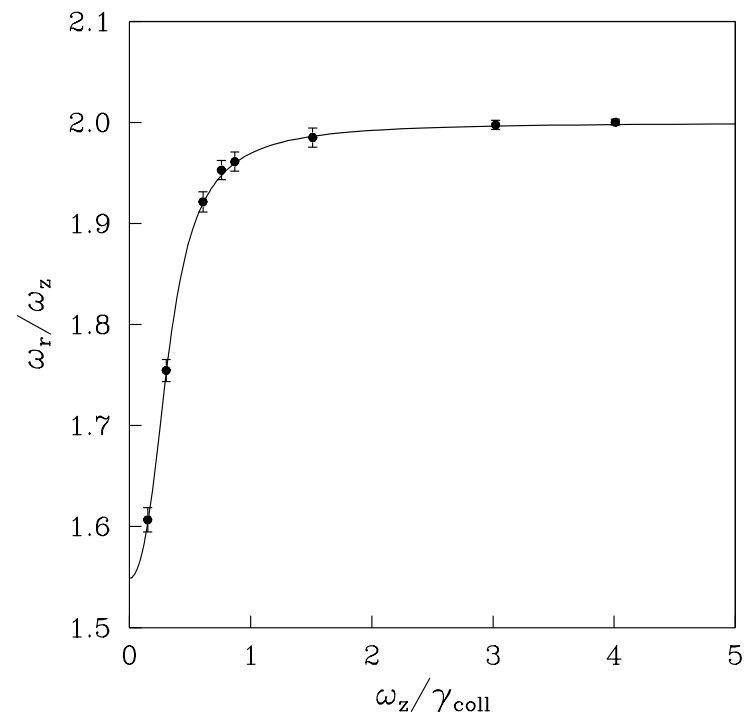

FIG. 1. Real part of frequency of the $l_{z}=0$ mode of a classical gas confined in a cigar shaped trap $(\lambda=1 / 10)$, versus $\omega_{z} / \gamma_{\text {coll }}$. The solid curve represents the prediction of the gaussian ansatz. The circles are the numerical results obtained with a moleclular dynamics simulation.

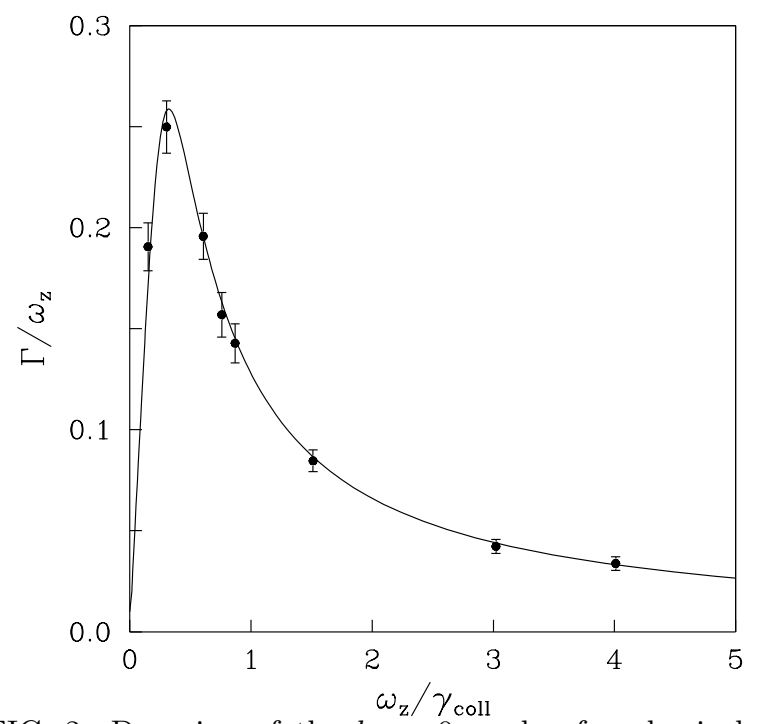

FIG. 2. Damping of the $l_{z} \stackrel{\omega_{z}}{=} 0$ mode of a classical gas confined in a cigar shaped trap $(\lambda=1 / 10)$, versus $\omega_{z} / \gamma_{\text {coll }}$. The notations for the line and the markers are the same as in figure 1. 